\title{
Public Health Genomics (PHG): From Scientific Considerations to Ethical Integration
}

\author{
YANICK FARMER \& BÉATRICE GODARD ${ }^{1}$
}

\begin{abstract}
Recent advances in our understanding of the human genome have raised high hopes for the creation of personalized medicine able to predict diseases well before they occur, or that will lead to individualized and therefore more effective treatments. This possibility of a more accurate science of the prevention and surveillance of disease also illuminates the field of public health, where the translation of genomic knowledge could provide tools enhancing the capacity of public health authorities to promote health and prevent diseases. But beyond scientific considerations, the use of genomics in public health research and interventions gives rise to several ethical and social issues of great importance. Considering the impact that PHG could have on the future of public health while still paying attention to the uncertainty surrounding the use of genomic databases for the benefit of populations, this article seeks to explore the promise of genomics in public health and the ethical issues that emerge from its application.
\end{abstract}

\section{Introduction}

Recent advances in our understanding of the human genome have raised high hopes for the creation of a personalized medicine able to predict diseases long before they occur, ${ }^{2}$ or that will produce more effective treatments. ${ }^{3,4}$ This possibility of a more efficient science of the prevention and surveillance of diseases also illuminates the field of public health, where genomic knowledge could provide tools enhancing the capacity of public health authorities to promote health and prevent diseases.

However, a major challenge for public health authorities will be the effective integration of genomic information into the public health response, whether it be for the identification/treatment of chronic diseases or even infectious ones that constitute a serious threat to populations and are costly for the health care system. In order to discover and characterize genes associated with diseases, as well as to understand how they interact with each other or with the environment to induce illness, some experts argue for the development of large biobanks that would gather biological materials, genetic, clinical and epidemiological information from large segments of the population. These data would facilitate the mapping of genetic variations occurring within specific populations and, along with other epidemiological and environmental information, could provide crucial insight into genetic susceptibilities and environmental threats.

Although genomics could initiate a transformation of the "one size fits all" paradigm in public health, ${ }^{5}$ there are concerns about "blind faith" in or overly optimistic views 
of scientific progress resulting from the most recent research in genomics and genetics. Some commentators argue that it is still premature to associate genetic variants with the expression of complex diseases. ${ }^{6}$ They argue that, given the interaction of the genetic and environmental factors behind complex diseases, the attempts to link relevant genes with the expression of the disease are invariably simplistic and offer little real hope for practical diagnostics and treatments.

Moreover, beyond scientific considerations, the use of biobanks and genomic databases for research and interventions in public health raise important ethical, legal and social issues. In addition to privacy concerns related to the use of personal data, public health genomics (PHG) deals with the traditional aspects of public health interventions, as well as with the fears of stigmatization and discrimination following a possible application of genomic tools within at-risk populations (eg, genetic screening).

Considering the impact that public health genomics could have for the future of public health, but in a context of significant uncertainty surrounding the use of genomic information to benefit populations, this article explores the promise of genomics in public health and the ethical issues emerging from the translation of genomic knowledge into public health interventions.

\section{The integration of genomics into public health}

The Human Genome Project ended in April 2003 with the expectation that important health benefits would result from current and future genomics research. But the growth in genomic knowledge, coupled with improvements in technical capacity (eg, bioinformatics), have been tempered by failures in the comprehension of complex diseases. ${ }^{7}$ Consequently, the promise of integrating genomics into public health has lead to scepticism.

\section{Possibilities of genomics research}

Many human diseases are the result of interactions between genetic variations and the environment. ${ }^{8}$ Genomics research is therefore essential for evidence-based disease prevention in a public health context. According to Brand and colleagues,

Nowadays, it is known that DNA determines not only the cause of single-gene disorders, which affect millions of people worldwide, but also predispositions ('susceptibilities'), which are based on genotype and haplotype variants, to common diseases. The new technologies will allow researchers to examine genetic mutations at the functional, genomic unit level, and to better understand the significance of environmental factors such as chemical agents, nutrition or personal behavior in relation to the etiology of cardiovascular diseases, allergies, cancer, psychiatric disorders or infectious diseases.

Advocates of public health genomics believe that genomics research will foster the development of new genomic tools applicable in a wide variety of public health 
settings. Genetic services related to maternal and child health (eg, prenatal and newborn screening) have been offered in many countries since the early $1960 \mathrm{~s},{ }^{10,11}$ and ongoing efforts and the increasing resources devoted to genomics could eventually pave the way for major advances in genetic screening, pharmacogenomics and other "-omics" technologies. ${ }^{12,13}$ In spite of their limits, ${ }^{14}$ technology using, for example, tandem mass spectroscopy or DNA microarrays are contributing to a finer understanding of the association between genetic variation and phenotypes, and are considered essential ways for elucidating the genetic determinants of health. With the help of these new technologies, genomics research could add to the traditional methods of risk assessment and contribute to the achievement of a new genomic medicine with significant impacts on public health. Genomics may allow clinicians or public health authorities to assess accurately an individual's risk and to recommend behaviours that should have positive effects on health. ${ }^{15}$

It is also expected than some genomics' applications, such as pharmacogenomics, will reduce the costs associated with the traditional "trial and error" method in curative medicine. ${ }^{16}$ At the population level, it could also play a substantial role in all aspects of public health (promotion, prevention, protection and surveillance). For instance, a profitable use of genetic testing, with the aim of providing an optimized and personalized drug therapy, can be accomplished by CYP2C9 genotyping. ${ }^{17}$ The allelic variants of this gene can identify individuals who show a higher risk of bleeding complications due to poor metabolizing of warfarin, an anticoagulant derived from coumarin. There are individual differences in response to anticoagulation therapy with warfarin derivates, and genotyping facilitates the administration of a therapy adapted to the patient's metabolism. Since anticoagulation therapies are indicated for several cardiovascular diseases such as congestive cardiomyopathies, atrial fibrillation, deep vein thrombosis or pulmonary embolism, the relevance of such a genomic screening method is obvious, both from personalized and public health perspectives.

The expected usefulness of genomics research is not limited to common or chronic diseases. Taking into account the damages provoked by tuberculosis, malaria, HIV and, more recently, by SARS, some studies have shown the potential of using genomic information to prevent pandemics of infectious diseases. ${ }^{18,19,20}$ In addition to improving knowledge of the pathogenesis of a disease, or of host-pathogen interactions, genomic profiling could help explain patterns of transmission and identify allelic variants responsible for disease propagation. Collecting data on genetic susceptibility and pathogens could subsequently lead to targeted interventions (eg, vaccines, surveillance) within at-risk populations. ${ }^{21}$

\section{Genomic databases}

Successful outcomes in genetic association studies of complex diseases hinge on the capacity to replicate results that initially seemed positive. Quite often, problems in identifying genetic aetiology of complex diseases are attributable to biological complexity and small samples with insufficient statistical power. ${ }^{22}$ This is one of the reasons why researchers in genomics have been calling for the creation of large-scale genomic databases. Several initiatives to collect genetic and phenotypic information for genomic research have been undertaken around the world (DeCode Genetics, 
Estonia Genome project, UK biobank, CARTaGENE, etc.). ${ }^{23,24,25}$ With this information, public health authorities would have the possibility of establishing evidence-based strategies to improve population health. ${ }^{26,27}$ The potential usefulness of these research infrastructures will to a large extent be determined by their functioning and by the nature of the data to which researchers will have access. The discoveries emerging from these data will have a deeper impact on public health if they are supplemented by other health information concerning, for instance, the lifestyles of individuals. The combining of data coming from genomic and other health databases will enable, just to give an example, a greater understanding of dietrelated diseases, linking genomic information on gene-food interactions with information about diet and individual lifestyles. ${ }^{28}$ In pharmacogenomics, the same method would apply to the creation of drug therapies combining clinical information with a patient's genomic profile.

Additionally, the SARS epidemic and concerns about a possible avian influenza pandemic have raised experts' awareness about the importance of implementing public health surveillance systems based on real-time epidemic detection and monitoring. ${ }^{29,30}$ A genetic vulnerability surveillance system using genomic and health databases could allow the detection, in real time or near-real time, of outbreaks of infectious and communicable diseases. In brief, whether it be for common diseases, drug therapies or infectious diseases, the use of genomic databases cannot be dissociated from public health genomics.

\section{The transfer of genomic knowledge in public health}

The translation of new findings in genomics into benefits for the population leads to the problem of the transfer of genomic knowledge in public health. Without close collaboration between genomics experts and public health policymakers it will be difficult to realize the promise of public health improvement by using genomic information.

In a recent article, Gwinn and Khoury identified three levels for the integration of genomics into public health. ${ }^{31}$ The first is related to the possibility of conducting population health research in genomics. As with genomic databases, the impact of new findings in genomics could be limited without large cohort studies confirming these findings and illustrating the risk factors related to specific diseases. The creation of large biobanks and databases, the use of existing samples or the exchange of data between researchers, as well as the collaboration of epidemiologists and geneticists, as it has been done at the U.S. Centers for Disease Control, ${ }^{32}$ would increase the impact of research in public health. South Korea, for instance, provides an example of successful exchanges between existing databases. The Korean Multi-center Cancer Cohort (KMCC) has collected data on lifestyle, physical activity, diet, reproductive factors and agricultural exposure. Moreover, biological materials (blood serum, plasma, buffy coat, packed erythrocytes) have been banked for future analysis. In 2004 , the KMCC and other Korean databases regrouped more than 80,000 subjects, and the collected data will allow public health authorities to identify risk factors for cancers, cardiovascular diseases and metabolic syndromes. ${ }^{33}$ 
Because of the difficulties of harmonization between epidemiological studies using large genomic databases, both international partnerships and the harmonization of parameters, measures and tests are seen as further necessary steps towards the integration of genomics in public health practices. ${ }^{34,35}$ It is considered that single cohort studies, even with large cohorts, more than likely have insufficient power to detect gene-environment interactions for numerous gene variants. As Wichmann and colleagues note, "The full potential of cohort studies to shed light on the occurrence of complex diseases will probably be realized only by pooling and synthesis across multiple populations with different genetic, environmental and sociocultural factors". ${ }^{36}$ Hence the creation of international initiatives, like the Public Population Project in Genomics $\left(\mathrm{P}^{3} \mathrm{G}\right),{ }^{37}$ regrouping biobanks and researchers from around the world.

The second level concerns the development of scientific evidence regarding the value of genomic information. This underlines the importance of disseminating information emerging from genomic research to ensure that public health stakeholders receive a clear demonstration of the clinical validity and utility of genomics. It implies that the education of public health professionals is essential to the success of public health genomics. ${ }^{38}$ Public health practitioners should have access to data and training on new genomic tools that could bring significant changes in public health. A number of recent technologies have proven their clinical value, but to be used properly, public health institutions need personnel trained in using sophisticated equipment. ${ }^{39}$ For this purpose, international networks are being developed to spread information about public health genomics and bring together people from different backgrounds that are interested in the possibilities of genomics in public health. HuGeNet ${ }^{40}$ is a good example of this kind of network. But these initiatives are still in their infancy and a lot has to be done to break down the barriers between genomic experts and their public health counterparts.

The third level refers to the integration of genomics into public health practice. According to Gwinn and Khoury, the realization of this process requires "careful policy planning and development that recognizes the complexity of genomics while building on approaches that have been successful in evaluating other health technologies". ${ }^{41}$ The development of an evidence-based decision making process is also a part of this third level of integration.

\section{Ethical issues}

In spite of its numerous expected advantages and its exponential scientific progress, public health genomics has raised a number of important ethical concerns. ${ }^{42,43}$ In fact, in order to keep its promise of improving public health, public health genomics needs to see its scientific progress harmonized with human dignity as expressed through adherence to fundamental ethical principles. The ethical issues related to the application of genomics in public health must therefore be examined. Some of these issues will be the same as those raised by genomics research in general. On the other hand, other ethical issues specific to public health will surface. 
The following sections emphasize the similarities and the differences between a more classical approach of the genomics-related ethical issues, which is based on Mendelian (monogenic) disorders, and a more contemporary (and relevant) approach that takes into account the biological complexity of polygenic disorders. Depending on the area of focus (eg, neonatal screening or genetic predispositions to complex diseases), an exhaustive ethical reflection on public health genomics should encompass both approaches.

\section{Genetic reductionism}

It has been reported that one of the first problem areas that comes to mind in the application of genomics toward health improvement is the reduction of human beings to their genes. ${ }^{44}$ In addition to the fatalism resulting from a vision where the genes are seen as a predominant health determinant, genetic reductionism leads to a major redefinition of public health. ${ }^{45}$ It can lead us to put the focus on genetic factors instead of also insisting on the importance of healthy behaviours and environments. It creates an "individualization" of responsibility ${ }^{46}$ in which individuals are carriers of inheritable "genetic defects" that represent a threat to public health. This situation might minimize the moral duty of the collectivity to provide equitable access to environmental health determinants such as healthy food, housing, education, etc. Genetic reductionism also results in a situation where individuals are experiencing a loss of hope in an open and undetermined future. It has been argued that the question of reductionism is largely outmoded since it is generally accepted that a majority of diseases are multifactorial, that is, they are the results of genetic and environmental factors. ${ }^{47}$ In fact, many highly prevalent diseases, such as type 2 diabetes, are also conditioned by environmental factors (eg, diet and lifestyle), which means that genetic testing only reveals predispositions instead of clear clinical diagnostic information. It is then important not to forget the other health determinants for preventing a fatalistic attitude from at-risk people. In that sense, public health genomics emphasises the complexity of biological processes, the importance of genegene and gene-environment interaction, and explicitly points out that its power lies in the application of mainstream public health interventions - healthy behaviours or environmental manipulation - but directed at sub-groups of the population segmented according to genetic risk. Genes are indeed a major health determinant but no more (or no less) major than environmental factors. Both contribute to disease risk.

\section{Protection of privacy}

Given that the integration of genomics into public health implies the use of genomic, phenotypic and epidemiological data, the questions linked to privacy and consent are at the centre of ethical reflection. First, by its nature, genomic information can help predict a person's medical future and divulge information about family members. For those reasons, genetic information is sometimes considered unique and deserving of special consideration, a view known as "genetic exceptionalism". 48,49

For many authors, a focus on genetic exceptionalism is not justified since the predictive value of genetic information is often limited, especially in the context of complex diseases. ${ }^{50}$ In addition, non-genetic biomarkers such as cholesterol level or blood pressure often have a greater predictive value than genotypic markers (except in 
the case of high penetrance inherited disorders). Consequently, for those authors, genetic information should receive the same level of protection as other health data. $^{51,52}$

Second, the complexity of privacy issues is also underscored by the possible commercialization of biological tissues, by the need to transfer or exchange information from one database to another and by the secondary use of data. ${ }^{53}$ Guidelines have been proposed over the last few years for ensuring the respect of human dignity and the protection of human rights and fundamental freedoms. ${ }^{54,55}$ Moreover, in order to maintain a balance between the protection of individual rights and the creation of biobanks and genomic databases as public goods, ${ }^{56,57}$ and in order to take into account the unforeseeable aspects of genomic research, different consent processes are proposed, but their scope and application is still subject to much debate. ${ }^{58,59}$

Third, the multiple potential uses of collected data seem to speak in favor of a broad consent instead of a specific one. This form of consent would include a right of withdrawal and transparency about the goals and the results of the research. ${ }^{60}$ This solution appears to be a good compromise between a "blanket consent" (unspecified uses of samples), which infringes on the right of the participant to be informed, and a strict notion of direct and ongoing informed consent, which is not always suitable for genomic research. ${ }^{61}$ The notions of "waivers" (alteration of some or all the elements of informed consent) and "multi-layered consent" have also been proposed to solve this delicate but fundamental problem. ${ }^{62}$ But even so, tension still persists between those who wish to reduce the formalities and facilitate research and those who wish to promote the right of the participant to be informed.

Privacy and confidentiality are closely linked to the level of consent. For instance, with anonymized or anonymous samples, it becomes possible to consider a broad consent, because the person is not identifiable. However some research projects require the linking of genetic information with clinical information, which is of the greatest importance for developing personalized treatment and prevention programs. Consequently, different coding procedures need to be developed to meet research objectives. ${ }^{63}$ Obviously, to ensure the smooth running of the identity protection process, special attention must be given to governance and supervision issues. ${ }^{64}$

\section{Stigmatization and discrimination}

The collection and storage of data for public health genomics opens individuals and communities to the threat of stigmatization and discrimination. One of the most common fears about genetic testing is the possibility of being penalized by insurance companies or by employers if individuals are found to be carriers of a genetic mutation. ${ }^{65,66}$ The potential discrimination differs from the one based on "visible" differences, but the logic is similar: to separate "good" genes from "abnormal" genes. ${ }^{67}$ In some of its applications (eg, genetic screening), this logic of separation could lead to a form of eugenics which might become socially acceptable because the selection would be made under the pretext of improving public health. 
The implementation, through public health genomics research, of more accurate methods of prevention and surveillance, to prevent infectious diseases for example, brings out the stigmatization and discrimination issues encountered in public health ethics. Which segment of the population will be subject to surveillance and on what grounds? Is there a risk of stigmatizing these sub-populations because of their genetic susceptibilities? Will the use of genomic technologies lead to new inequalities among people? Even if the risks of stigmatization or discrimination might be exaggerated, they might have negative impacts on research, especially on the recruitment of subjects and the fears might prevent the development of public health genomics and its benefits to populations. Such questions underline the importance, for public health genomics, of not loosing sight of the ethical principles at the root of public health ethics. ${ }^{68,69,70}$ Yet, the literature is sparse that addresses public health genomics and public health ethics. Articles on the ethical aspects of genomics are more concerned with research ethics, while articles on public health ethics present problems related to traditional approaches to public health.

\section{Individual versus collective rights}

In an era of heightened human rights awareness, the use of public health authorities' power is becoming more and more complicated. ${ }^{71}$ This also holds for pubic health genomics. The use of genomics as a privileged tool of research, prevention, protection or surveillance in a public health context provides an example of a classic problem in public health ethics: the respect of both individual and collective rights.

On the one hand, there is the obligation to respect individuals' rights based on their autonomy and privacy, whether it be in the collection of biological materials and data or in the implementation of measures for improving public health. On the other hand, there is the right of the whole population to be protected against environmental threats, which might imply, in extraordinary situations, the application of coercive measures. ${ }^{72}$

Public Health Genomics (PHG), with its actions undertaken at the population level, necessitates the emergence of an ethical paradigm that goes beyond the clinical and individual-based principle of autonomy. ${ }^{73}$ It has been suggested that, in public health interventions, the precautionary principle may serve as the starting point of a new ethics of risk management, ${ }^{74,75}$ which could be applicable to PHG. Ethical rules inspired by John Stuart Mill's harm principle ${ }^{76}$ limit preventive interventions to a minimum in order to protect individuals against the violation of their rights. Interventions are justified only if the risk is significant. But according to the precautionary principle, there is an "obligation to protect populations against reasonably foreseeable threats, even under conditions of uncertainty" ${ }^{77}$ Thus, the precautionary principle highlights the potential harm of inaction and points to the importance of scientific assessment of risk. This interventionist approach to public health was applied in Europe when cases of mad cow disease were reported. ${ }^{78}$ Given the added value that risk management based on the study of gene-environment interactions represents, and since an individualistic vision of autonomy could put a brake on the evolution of PHG, the precautionary principle is seen as part of a 
solution, bridging the gap and easing the tension between individual and collective rights.

The concept of "public good" might also be a part of the solution to this ethical dilemma. ${ }^{79}$ It stipulates that in an era of liberalism and globalization, the creation of public goods compensates for the inequalities resulting from an inequitable distribution of genomic technologies. "Global public goods are defined as goods that are both non-competitive and non-exclusive. They are, in other words, enjoyable by all without detriment to others. ${ }^{, 80}$ To some experts, genomic databases correspond to the definition of a public good because they are universally accessible resources. They belong to the common heritage of mankind and should therefore not be patented for private use. ${ }^{81}$ In this perspective, by promoting a "common ownership" of genomic knowledge, by improving public health and by tailoring interventions for communities, the creation of genomics research infrastructures could be perceived as a public goods that respond to the most fundamental needs of individuals (eg, equity, beneficence) while achieving the goals of the community. ${ }^{82}$

\section{Benefit-sharing}

To the extent required by their status of public resource, there is a moral obligation to return to the population, at least partially, the benefits yielded by genomics research infrastructures. ${ }^{8,84}$ Benefit-sharing can take various forms ${ }^{85}$ : research on conditions or issues that are important to the community, training for local researchers, providing access to information and services generated by genomics research, free or reducedprice access to tests or treatments developed through genomics research, and creation of local infrastructure and employment opportunities, etc.

In an international perspective, a transfer of knowledge and technologies to developing countries could be proposed to reduce social and economic inequalities among nations. Whatever form it takes, benefit-sharing should be supported by a clear ethical vision. Should benefit-sharing be motivated by solidarity with vulnerable communities? Should we consider the idea of sharing financial benefits? Or should only health benefits be shared?

In the absence of a clear ethical vision about benefit-sharing, participation in research projects involving genomic databases might be compromised, and eventually, concrete and tangible benefits of public health genomics for the directed population might be questioned.

\section{Public participation}

Because citizens have been or will be solicited as research subjects, because they support the risks of genomics research as well as benefit from its results, and because of the power imparted to public health authorities, the ethical integration of genomics into public health requires the informed awareness of lay citizens, and eventually their participation in the formulation of public policy. Otherwise, the situation might evolve toward the creation of social inequalities or cause undesirable harms. Growing disparities between experts and non-experts are contrary to the ideal of transparency. Consequently, great attention should be paid to the role of the public in PHG. Most 
genetics experts recognize the importance of public awareness. ${ }^{86,87,88}$ Great attention is accorded to informing the public, but this often leads to a one-sided dialogue that doesn't afford lay citizens much influence in policy-making. ${ }^{89,90}$ Increasingly, however, voices are emerging on the theme of public participation in biotechnology policy-making. ${ }^{91,92,93}$ From consensus conferences to citizens' workshops, various mechanisms have been set up to increase the influence of the lay citizenry. Still, even if the idea of enrolling citizens is in keeping with democratic values, many questions remain. First, public participation models are expensive and their organization is very time-consuming. Do the results make up for the costs? Second, given the complexity of genomics-related issues, lay citizens must be trained by experts to facilitate the debate with other experts. Is there a risk of being biased? Third, to be democratic, these mechanisms must be representative. How can this representativeness be attained and thereafter evaluated?

These questions show that the efficacy and efficiency of public participation models must be fully thought through. From the diversity of citizens' personal experiences a "popular wisdom" could emerge and represent an added value for policy-making in PHG. But the extent to which the public should be engaged remains an unsolved problem, one that must further be examined.

\section{Conclusion}

Scientific research using genomic infrastructures aims to offer new opportunities to improve public health. Recent advances in pharmacogenomics suggest that it will be possible to apply personalized treatments adapted to the genomic profile of each patient. The idea of a new genomic medicine provides hope for an improved and more cost-effective management of disease.

The implementation of genomic-based strategies could also bring a significant contribution to the redefinition of public health. The translation of genomic knowledge into clinical practice reveals the possibility of changing not only the way research in public health is conducted, but also how subsequent interventions may be carried out. For instance, the prevention of chronic diseases and the promotion of healthy behaviours could be facilitated by the predictive capacity of more sophisticated genetic tests. Furthermore, the creation of databases containing genomic information on populations and on pathogens, supported by new data processing tools, could help redesign surveillance systems in which outbreaks of infectious diseases would be detected earlier than with traditional methods.

However, technical, economic, legal and ethical barriers may hinder the advent of public health genomics. The realization of the full potential of PHG is still years ahead and the investments needed are substantial. The social costs of potential discrimination, stigmatization or invasion of privacy add further obstacles. The longterm development of PHG will require an appropriate integration of the scientific, economic and ethical dimensions. Public participation might also contribute to the integration process. Considering that, ultimately, the whole population will have, at 
least in part, to support the economic and social costs of PHG, it is therefore crucial to take the views of the public fully into account.

To date, little has been done to help PHG stakeholders (genomics and public health scientists, policy-makers, health care providers, etc.) to ethically integrate genomics into public health planning. Many grey areas subsist around those ethical principles that should be applied to PHG (eg, consent, secondary use of data). That is why major fieldwork should be undertaken with stakeholders as well as the public, to obtain a wide range of views and opinions, which could lead to the development of a suitable ethical framework and to appropriately tailored public policies.

\section{Acknowledgments}

This article has benefited from the financial support of Genome Canada and Genome Quebec, through the project Genomics and public health: building public "goods"? We thank Bryn Williams-Jones and Philippe-Aubert Côté for their helpful comments.

\footnotetext{
${ }^{1}$ The authors are at Programmes de bioéthique, Université de Montréal, Montréal, Canada. Correspondence to beatrice.godard@umontreal.ca.

${ }^{2}$ J. Bell. Predicting disease using genomics. Nature 2004; 429: 453-56.

${ }^{3}$ W. Kalow. Pharmacogenetics and personalized medicine. Fundamental and Clinical Pharmacology 2002; 16: 337-42.

${ }^{4}$ W.E. Evans and M.V. Relling. Moving towards individualized medicine with pharmacogenomics. Nature 2004; 429: 464-68.

${ }^{5}$ M. Gwinn and M.J. Khoury. Research Priorities for Public Health Sciences in the Postgenomic Era. Genetics in Medicine 2002; 4 (6): 410-11.

${ }^{6} \mathrm{H}$. Colhoun et al. Problems of reporting genetic associations with complex outcomes. Lancet 2003; 361: 865-72.

${ }^{7}$ G. Davey Smith et al. Genetic epidemiology and public health: hope, hype and future prospects. Lancet 2005; 366: 1484-98.

${ }^{8}$ M.J. Khoury et al. (eds.) 2000. Genetics and public health in the 21st century: Using genetic information to improve health and prevent disease. New York: Oxford University Press.

${ }^{9}$ A. Brand et al. Getting ready for the future: integration of genomics into public health research, policy and practice in Europe and globally. Community Genetics 2006; 9: 67-71.

${ }^{10}$ D. Alexander et al. A vision of the future of newborn screening. Pediatrics 2006; 117: 350-4.

${ }^{11}$ N.A. Holtzman. What role for public health in genetics and vice versa?. Community Genetics 2006; 9: 8-20.

${ }^{12}$ K. Ries Merikangas and N. Risch. Genomic Priorities and Public Health. Science 2003; 302: 599601.

${ }^{13}$ K. Freeman. Harnessing the HGP for public health. Environmental Health Perspectives 2004; 112 (7): 403.

${ }^{14} \mathrm{~K}$. Gunderson et al. A genome-wide scalable SNP genotyping assay using microarray technology. Nature Genetics 2005; 37: 549-554.

${ }^{15} \mathrm{~S}$. Shostak. Locating gene-environment interaction: at the intersection of genetics and public health. Social Science \& Medicine 2003; 56: 2327-2342.

${ }^{16}$ J. Dausset. Les bases de la médecine prédictive. ADSP (Haut comité de la santé publique) 2001; 37 : 66-67.

${ }^{17}$ E. Kunstmann and J.T. Epplen. Genetic counseling for the public? Community Genetics 2006; 9: 6266.
} 
${ }^{18}$ J. Ziebuhr. Molecular Biology of Severe Acute Respiratory Syndrome Coronavirus. Current Opinion in Microbiology. August 2004; 7 (4): 412-9.

${ }^{19}$ R.E. Johnston. A Candidate Vaccine for Severe Acute Respiratory Syndrome. The New England Journal of Medicine 2004; 351 (8): 827-8.

${ }^{20} \mathrm{R}$. Freeman et al. Use of rapid genomic deletion typing to monitor a tuberculosis outbreak within an urban homeless population. Journal of Clinical Microbiology. 2005; 43 (11): 5550-54.

${ }^{21}$ A.J. Frodsham and A.V.S. Hill. Genetics of infectious diseases. Human Molecular Genetics 2004; 13 (2): 187-94.

${ }^{22}$ Davey Smith, op. cit. note 7.

${ }^{23}$ M.J. Khoury et al. The emergence of epidemiology in the genomics age. International Journal of Epidemiology 2004; 33: 936-44.

${ }^{24}$ K.J. Maschke. Navigating an ethical patchwork - human gene banks. Nature Biotechnology 2005; 23 (5): 539-45.

${ }^{25}$ J. Kaiser. Biobanks. Population databases boom from Iceland to the U.S. Science 2002; 298 (5596): $1158-61$

${ }^{26}$ Gwinn \& Khoury, op. cit. note 5.

${ }^{27}$ M.J. Khoury et al. Population Screening in the Age of Genomic Medicine. New England Journal of Medicine 2003; 348 (1): 50-58.

${ }^{28}$ M. Müller et al. Nutrigenomics: goals and strategies. Nature Reviews Genetics 2003; 4: 315-22.

${ }^{29}$ Y.H. Hsieh et al. Real-time forecast of multiphase outbreak. Emerging Infectious Diseases 2006; 12 (1): 122-7.

${ }^{30}$ E. Mykhaloskiy et al. The Global Public Health Intelligence Network and early warning outbreak detection: a canadian contribution to global public health. Canadian Journal of Public Health 2006; 97 (1): $42-4$.

${ }^{31}$ M. Gwinn and M.J. Koury. Genomics and Public Health in the United States: Signposts on the Translation Highway. Community Genetics 2006; 9: 21-26.

${ }^{32}$ Ibid.

${ }^{33}$ K.Y. Yoo et al. Genomic epidemiology cohorts in Korea: present and the future. Asian Pacific Journal of Cancer Prevention 2005; 6 (3): 238-43.

${ }^{34}$ M.J. Khoury. The case for a global human genome epidemiology initiative. Nature Genetics. 2004; 36 (10): 1027-28.

${ }^{35}$ H.E. Wichmann et al. KORA-gen. Resources for population genetics. Controls and a broad spectrum of disease phenotypes. Gesundheitswesen 2005; 67 (1): 26-30.

${ }^{36}$ Khoury, op. cit. note 34 .

${ }^{37}$ Wichmann et al, op. cit. note 35 .

${ }^{38}$ A.S. Noonan. Integrating genomics into US public health. Genetics in Medicine 2002; 4 (6): 68S$71 \mathrm{~S}$.

${ }^{39}$ B.H. Robertson et al. New microbiology tools for public health and their implications. Annual Review of Public Health 2005; 26: 281-302.

${ }^{40}$ Human Genome Epidemiology Network www.cdc.gov/genomics/hugenet/default.htm (accessed 31 August. 2006)

${ }^{41}$ Gwinn \& Khoury, op. cit. note 31.

${ }^{42}$ R. Chadwick et al. Solidarity and equity: new ethical frameworks for genetic databases. Nature Reviews Genetics 2001; 2 (4): 318-321.

${ }^{43}$ F.S. Collins. The case for a US prospective cohort study of genes and environment. Nature 2004; 429: 475-77.

${ }^{44}$ I. Rabino. Genetic Testing and Its Implications: Human Genetics Researchers Grapple with Ethical Issues. Science, Technology \& Human Values 2003; 28 (3): 365-402.

${ }^{45}$ Shostak, op. cit. note 15 .

${ }^{46} \mathrm{~W}$. van den Daele. The spectre of coercion: is public health genetics the route to policies of enforced disease prevention? Community Genetics 2006; 9: 40-49.

${ }^{47}$ M.H.V. Van Regenmortel. Reductionism and complexity in human molecular biology. Embo Report. 2004; 5 (1): 1016-1020. 
${ }^{48}$ T.H. Murray. 2000. Genetic Exceptionalism and 'Future Diaries': Is Genetic Information Different from Other Medical Information?, in Genetic Secrets: Protecting Privacy and Confidentiality in the Genetic Era. M.A. Rothstein, ed. New Haven: Yale University Press.

${ }^{49}$ J. Hodge. Ethical Issues Concerning Genetic Testing and Screening in Public Health. American Journal of Medical Genetics Part C 2004; 125C: 66-70.

${ }^{50}$ F.S. Collins et al. Heredity and Humanity: Have no fear. Genes aren't everything. The New Republican 2001; June 25: 27-29.

${ }^{51}$ Murray, op. cit. note 48.

${ }^{52}$ A.W. Toga and P.M. Thomson. Genetics of brain structure and intelligence. Annual Review of Neuroscience 2005; 28: 1-23.

${ }^{53}$ A. Cambon-Thomsen et al. Populational Genetic Databases : Is a Specific Ethical and Legal Framework Necessary? GenEdit 2005; 3 (1): 1-13.

${ }^{54}$ UNESCO. 2003. International Declaration on Human Genetic Data. Paris. October 16.

${ }^{55}$ UNESCO. 1997. Universal Declaration on the Human Genome and Human Rights. Paris. November 11.

${ }^{56} \mathrm{H}$. Thortsteindottir et al. Genomics. a global public good? Lancet 2003; 361 (9361): 891-92.

${ }^{57}$ B.M. Knoppers. Of Genomics and Public Health: Building Public Goods? CMAJ 2005; 173 (10): 1185-86.

${ }^{58}$ T. Caufield et al. DNA databases and consent: a suggested policy option involving an authorization model. BMC Medical Ethics 2003; 4 (1): 1-4.

${ }^{59}$ B.M. Knoppers. Consent revisited: points to consider. Health Law Review 2005; 13 (2-3): 33-38.

${ }^{60}$ Ibid.

${ }^{61} \mathrm{~V}$. Arnason. Coding and consent: moral challenges of the database project in Iceland. Bioethics 2004; 18 (1): 27-49.

${ }^{62}$ B.S. Elger et al. Consent and anonymization in research involving biobanks: differing terms and norms present serious barriers to an international framework. EMBO reports 2006; 7 (7): 661-6.

${ }^{63}$ B.M. Knoppers et al. The Babel of genetic data terminology. Nature Biotechnology 2005; 23 (8): 925-7.

${ }^{64}$ Cambon-Thomsen, op. cit. note 53.

${ }^{65}$ E.W. Clayton. Ethical. legal and social implications of genomic medicine. The New England Journal of Medicine 2003; 349: 562-9.

${ }^{66}$ Y. Joly et al. Genetic information and life insurance: a 'real' risk?. European Journal of Human Genetics 2003; 11 (8): 561-4.

${ }^{67}$ M.A. Rothstein. What is genetic discrimination and when and how can it be prevented? Genetics in Medicine 2001; 3 (5): 354-8.

${ }^{68}$ R. Bayer et al. The Genesis of Public Health Ethics. Bioethics 2004; 18 (6): 473-492.

${ }^{69}$ J.F. Childress et al. Public Health Ethics: Mapping The Terrain. Journal of Law, Medicine \& Ethics 2002; 30 (2): 171-8.

${ }^{70}$ D. Callahan et al. Ethics and Public Health: Forging a Strong Relationship. American Journal of Public Health. 2002; 92 (2): 169-76.

${ }^{71}$ A. Harris et al. The Exercise of Public Health Powers in an Era of Human Rights: the Particular Problems of Tuberculosis. Journal of the Royal Institute of Public Health 2004; 118: 313-22.

${ }^{72}$ van den Daele, op. cit. note 46.

${ }^{73}$ O. O’Neill. Public Health or Clinical Ethics: Thinking Beyond Borders. Ethics and International Affairs. 2004; 16 (2): 35-45.

${ }^{74}$ Bayer, op. cit. note 68 .

${ }^{75}$ R. Andorno. The Precautionary Principle: A New Legal Standard For a Technological Age. Journal of International Biotechnology Law. 2004; 1: 11-19.

76 "The only purpose for which power can be rightly exercised over any member of a civilized community against his will is to prevent harm to others." - John Stuart Mill. On liberty.

${ }^{77}$ Bayer, op. cit. note 68.

${ }^{78}$ R. Massé. 2003. Éthique et santé publique. Enjeux, valeurs et normativité. Québec. Presses de l'Université Laval.

${ }^{79}$ I. Kaul. I. Grunberg. I. and M.A. Stern, eds. 1999. Global Public Goods. New York. Oxford University Press. 
${ }^{80}$ R. Chadwick. Nutrigenomics, individualism and public health. Proceedings of Nutrition Society 2004; 63: 161-66.

${ }^{81}$ HUGO. 2002. HUGO Ethics Committee Statement on Genomic Databases. London. December.

${ }^{82}$ R. Chadwick and S. Wilson. Genomic databases as global public goods?. Res Publica 2004; 10 (2): 123-34.

${ }^{83}$ Natural Sciences and Engineering Research Council of Canada (NSERC). Medical Research Council of Canada (MRC). Social Sciences and Humanities Research Council of Canada (SSHRC). 1998. TriCouncil Policy Statement: Ethical Conduct for Research Involving Humans. Ottawa.

${ }^{84}$ B. Godard et al. Data storage and DNA banking for biomedical research: informed consent. confidentiality. quality issues. ownership. return of benefits. A professional perspective. European Journal of Human Genetics 2003; December 11. S2: S82-S122.

${ }^{85} \mathrm{~J}$. Tansey et al. 2004. The foundations, applications and ethical dimensions of biobanks. Electronic Working Papers Series. W. Maurice Young Center for Applied Ethics. University of British Columbia. ${ }^{86}$ Brand et al, op. cit. note 9.

${ }^{87}$ Human Genome Epidemiology Network, op. cit. note 40.

${ }^{88}$ The Economic and Social Research Council (ESRC). 2005. Science, society and public policy: dilemmas of public participation. Royal Society. London. 25 June. www.genomicsforum.ac.uk/documents/PDF/ScienceSocietyandPublicPolicyMeetingReport.pdf (accessed 2 August. 2006).

${ }^{89}$ A. Thibault et al. 2000. Cadre de référence de la participation publique (démocratique. utile et crédible). Conseil de la santé et du bien-être. Quebec.

${ }^{90}$ L. Turnbull et al. 2006. Fostering Canadians' Role in Public Policy: A Strategy for Institutionalizing Public Involvement in Policy. Canadian Policy Research Networks. Ottawa. www.cprn.com/fr/doc.cfm?doc $=1404$ (accessed 2 August. 2006).

${ }^{91}$ J. Chopyak et al. Public participation in science and technology decision making: trends for the future. Technology in Society. 2002; 24: 155-66.

92 M.M. Burgess. Public Consultation in Ethics. An Experiment in Representative Ethics. Journal of bioethical inquiry. 2003; 1 (1): 4-13.

${ }^{93}$ Abelson. J. et al. Will it make a difference if a show up and share? A citizens perspective on improving public involvement processes for health system decision-making. Journal of Health Services. Research \& Policy. 2004; 9 (4): 205-12. 